\section{Queja por plagio de artículo y solicitud de acciones correctivas}

Victoria E. Díaz Facio Lince ${ }^{1}$

Cristian David Osorio Yepes ${ }^{1}$

${ }^{1}$ Universidade de Antioquia. Antioquia Colombia.

Queremos manifestar ante ustedes un caso de plagio en un artículo publicado en su revista, en el cual consideramos que los autores Roberto Ariel Abeldaño y Ruth Fernández infringen las normas internacionales de los derechos de autor, con respecto a que:

1. Nuestro artículo "Modelos de intervención psicosocial en situaciones de desastre por fenómeno natural"' fue publicado en la Revista de Psicología de la Universidad de Antioquia, volumen 4, número 2, en el año 2012: http://aprendeenlinea. udea.edu.co/revistas/index.php/psicologia/article/ view/15481

2. El artículo "Salud mental en la comunidad en situaciones de desastre. Una revisión de los modelos de abordaje en la comunidad"2, fue publicado por su editorial, revista Ciência \& Saúde Coletiva, volumen 21, número 2, en el año 2016. Doi:10.1590/1413-81232015212.175020.

3. Si bien nuestro artículo se cita de manera directa en algunos párrafos del artículo en mención, es de considerar que múltiples ideas alrededor de dichas citas aparecen como propiedad de Abeldaño y Fernández ${ }^{2}$. Por ejemplo, en el apartado Modelos de intervención psicosocial en desastres en América Latina, se lee:

En los últimos años, a partir de un cambio de paradigma, se realiza énfasis en una concepción de salud mental integral, pasando de lo individual a lo colectivo y de considerar el déficit a las potencialidades de la comunidad ${ }^{37}$.

En general, pueden clasificarse los modelos de intervención entre los que están basados en el tiempo, intervenciones centradas en tipos de eventos específicos, las intervenciones que se realizan por niveles de acción y las intervenciones centradas en las personas ${ }^{2}$ (Abeldaño y Fernández, 2016, p. 437).

(Nota: Los fragmentos que ponemos en itálico son apartados textuales de nuestro artículo).

Igualmente, en las discusiones:

Así también, el presente trabajo revisó una serie de modelos de abordaje de la salud mental en situaciones de desastre. Se incluyeron varias perspectivas para el abordaje de la salud mental comunitaria en esas situaciones: las intervenciones basadas en el tiempo, las que están centradas en un tipo de desastres específico, las intervenciones por niveles de acción o esferas y las centradas en las personas ${ }^{2}$ (Abeldaño y Fernández, 2016, p. 439).

Nótese que la clasificación de modelos de intervención basados en el tiempo, intervenciones por niveles de acción e intervenciones centradas en las personas, es tomada directamente del artículo de Osorio y Díaz (2012)1 , lo cual no se dice explícitamente. Lo anterior deja la idea de que es propuesta de Abeldaño y Fernández $(2016)^{2}$. Y más adelante, se lee:

Intervenciones basadas en el tiempo. En este modelo, las intervenciones ponen énfasis en la cuestión temporal: antes, durante y después del desastre. Los intereses son prioritariamente puestos en las respuestas desde dos puntos de vista: las consecuencias de la población frente al desastre y las acciones que pueden realizar los profesionales con esa comunidad ${ }^{2}$ (Abeldaño y Fernández, 2016, p. 437).

Se extrae, por tanto, la misma clasificación e ideas del artículo de Osorio y Díaz (2012), con otras palabras, sin dar crédito a los autores originales:

Con respecto a las intervenciones que hacen énfasis en el tiempo - antes, durante y después del desastre -, se observó un interés en desligarse de la afectación material, basándose en la respuesta desde dos puntos de vista temporales: por un lado, dando prioridad a las reacciones o consecuencias de la población frente al desastre; y por el otro, a las acciones que pueden realizar los profesionales desde un marco institucional" ${ }^{1}$ (Osorio y Díaz, 2012, p. 73-74).

Asimismo, en este caso se hace un resumen de nuestro apartado "2.2.2. Intervenciones por niveles de acción o esferas", con la misma fuente (50: proyecto Esfera) y sin hacer referencia a nuestra autoría:

Intervenciones por niveles de acción o esferas. En este caso las intervenciones pasan de estar centradas en la supervivencia y la recuperación material a estar basadas en niveles integrados dependiendo de las necesidades de la población afectada y contemplando los distintos sectores de acción. Aquí cobra gran relevancia la capacidad de articular a la comunidad afectada y demás actores sociales a los procesos que llevan a cabo distintas instituciones, con el objetivo de resolver necesidades de distintos niveles en una situación de desastre. El Proyecto Esfera $^{50}$, aunque su sede se encuentra en Ginebra, Suiza, representa una iniciativa internacional que dio los primeros pasos en implementar estrategias integrales en situaciones de desastre ${ }^{2}$ (Abeldaño y Fernández, 2016, p. 437).

Nótese la relación: 
Se evidenció un paso de las intervenciones centradas en la supervivencia y la recuperación material a intervenciones basadas en niveles, las cuales consisten en una serie de acciones mínimas e integrales, desde distintos sectores de la ayuda humanitaria, hacia distintos tipos de necesidades de la población afectada.

Esto ha significado una integración de profesionales y de esfuerzos articulados a las necesidades concretas de la población afectada por cada desastre. De este modo, la estrategia que se debe llevar a cabo es la capacitación de cada grupo interviniente, sobre el impacto y la evaluación de las necesidades de atención a las poblaciones en materia de salud mental y apoyo psicosocial.

La integralidad en las intervenciones está relacionada con acciones realizadas desde dos puntos de vista: 1) Interno: la organización de diversas instituciones y profesionales en función de resolver múltiples daños e impactos en situaciones de desastre. 2) Externo: la capacidad de articular a la comunidad afectada y demás actores sociales a los procesos liderados por diversas instituciones, con el objetivo de resolver sus múltiples necesidades en una situación concreta de desastre.

El Proyecto Esfera (SCHR, VOICE, ICVA, 2004) fue uno de los programas pioneros para impulsar acciones humanitarias e intersectoriales en situaciones de desastre (Osorio y Díaz, 2012, p. $74-75)^{1}$.

4. Algunos párrafos toman otras ideas de nuestro artículo que son redactadas como propias por los autores Abeldaño y Fernández².

Por ejemplo, en el apartado Discusiones:

La mayoría de los documentos analizados están orientados a los profesionales intervinientes y son escasos los documentos rescatados que sugieran intervenciones con participación comunitaria como herramienta fundamental (más allá de las recomendaciones de los organismos internacionales), esto sugiere la necesidad de generar y apoyar programas donde el empoderamiento y la participación comunitaria sean el eje de las intervenciones psicosociales (Abeldaño y Fernández, 2016, p. 439)2.

Léase en las conclusiones de nuestro artículo:

La mayoría de los documentos analizados están orientados a los profesionales intervinientes. Esto habla de la necesidad de dirigir más herramientas, incluidas la retroalimentación de las lecciones aprendidas, a las poblaciones en condiciones de alta vulnerabilidad. Es allí donde un tercer tipo de enfoque en las intervenciones ha cobrado gran fuerza: la participación comunitaria" (Osorio y Díaz, p. 80)1.
De igual manera, en el apartado Modelos de intervención psicosocial, se lee:

Existen diversas perspectivas y modelos de intervención psicosocial en situaciones de desastre descriptas en la literatura, en base a las experiencias internacionales. Algunos se basan en enfoques de desarrollo humano, biológico-epidemiológico o comunitario, a partir de lo que se concibe como salud mental y de la concepción del término "desastre". En los últimos años, a partir de un cambio de paradigma, se realiza énfasis en una concepción de salud mental integral, pasando de lo individual a lo colectivo y de considerar el déficit a las potencialidades de la comunidad ${ }^{37}$ (Abeldaño y Fernández, 2016, p. 437) ${ }^{2}$.

Si bien en este párrafo se cita nuestro artículo de manera directa (no.37), lo que señalamos en negrilla también es tomado del artículo original sin la respectiva cita, o no citado como lo establecen las normas:

En términos generales se encontraron múltiples perspectivas en la concepción de lo psicosocial y la salud mental en relación con los desastres. La mayoría de ellas se limitan a una mirada pragmática que no ahonda en la conceptualización. Gran cantidad de modelos utilizan, sin definir, los términos psicosocial y salud mental.

Los enfoques de salud mental encontrados en las unidades documentales se basaron en modelos de desarrollo humano, biológicos-epidemiológicos y comunitarios. En la última década se ha hecho énfasis en un concepto de salud mental integral que ha pasado de lo individual a lo colectivo y del déficit a las potencialidades (Osorio y Díaz, P. 72) ${ }^{1}$.

Por las razones anteriores, y toda vez que todo plagio atenta contra la ética que nuestras disciplinas promulgan así como contra los derechos de autor, solicitamos comedidamente:

1. Se verifiquen estas pruebas por parte del Consejo editorial de la revista Ciência \& Saúde Coletiva (ver archivos con artículos completos adjuntos).

2. Una vez establecido lo anterior, se desmonte el artículo de las publicaciones de la revista, se exija el retracto por parte de los autores implicados y se nos notifique por medio de un comunicado oficial.

3. Asimismo, solicitamos enviar copia de dicho comunicado a las bases de datos o los medios de difusión científica en los que se encuentre dicho artículo; en nuestra pesquisa pudimos establecer que está publicado en:

Scielo (castellano):

http://www.scielo.br/scielo.php?script=sci_ arttext\&pid=S1413-81232016000200431

PubliMed (inglés): 
https://www.ncbi.nlm.nih.gov/pubmed/269 10151

Redalyc (castellano):

http://www.redalyc.org/html/630/630441 57011/index.html

The free library (inglés):

https://www.thefreelibrary.com/Community + mental+health+in+disaster+situations. $+\mathrm{A}+$ review+of...-a0451532197

Dimensions:

https://app.dimensions.ai/details/publication/ pub.1020706281

\section{Adjuntos}

-Artículo: Salud mental en la comunidad en situaciones de desastre. Una revisión de los modelos de abordaje en la comunidad (Abeldaño y Fernández, 2016) Ver subrayados en amarillo y notas de plagio al hacer click en ellos.

- Artículo: Modelos de intervención psicosocial en situaciones de desastre por fenómeno natural (Osorio y Díaz, 2012)

Agradecemos su diligencia y oportunas acciones frente a las solicitudes de este caso.

\section{Referências}

1. Osorio Yepes CD, Diaz Facio Lince VE. Modelos de intervención psicosocial en situaciones de desastre por fenómeno natural. Revista de Psicología de la Universidad de Antioquia 2012; 4(2):65-84.

2. Abeldaño RA, Fernández R. Salud mental en la comunidad en situaciones de desastre. Una revisión de los modelos de abordaje en la comunidad. Cien Saude Colet 2016; 21(2):431-442. 
